\title{
Determination of Germination Parameters of Safflower (Carthamus tinctorius L.) Cultivars Under Salt Stress
}

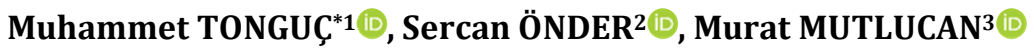 \\ 1,2Isparta University of Applied Science, Faculty of Agricultural, Department of Agricultural Biotechnology, \\ 32200, Isparta, Turkey \\ ${ }^{3}$ Isparta University of Applied Science, Faculty of Agricultural, Department of Field Crops, 32200, Isparta, Turkey
}

(Alınış / Received: 15.06.2021, Kabul / Accepted: 16.07.2021, Online Yayınlanma / Published Online: 15.08.2021)

\section{Keywords}

Germination indices, Germination stress index, Abiotic stress

\begin{abstract}
The present study was carried out to characterize germination capacity of 10 safflower cultivars under saline conditions. Five salt $(\mathrm{NaCl})$ levels $(0,60,120$, $180,240 \mathrm{mM}$ ) were used to test safflower seeds. Germination of seeds was counted every day for 14 days and germination percentage, Timson's germination index, mean germination time, mean germination rate and germination stress index were calculated. Cultivar and salinity treatments were important for all parameters; however mean germination time and mean germination rate interactions were not important. Germination percentage, Timson's germination and germination stress indices decreased significantly with increased salt concentrations. However, mean germination time increased with higher salt concentrations. The most significant reductions in germination percentage were observed at 180 and $240 \mathrm{mM}$ salt concentrations. Correlation coefficients were all important for germination indices. Based on germination indices, Leed and FO2 were more sensitive to salt stress at germination stage and Royal was more resistant than the other cultivars tested. Germination percentage, Timson's germination index and germination stress index were better to assess germination capacity of safflower cultivars under salt stress conditions. Mean germination time and rate were not suitable to assess differences in germination parameters of the cultivars under stress conditions.
\end{abstract}

\section{Aspir (Carthamus tinctorius L.) Çeşitlerinin Tuz Stresi Altinda Çimlenme Parametrelerinin Belirlenmesi}

Anahtar Kelimeler

Çimlenme endeksleri, Çimlenme stres indeksi, Abiyotik stres
Özet: $\mathrm{Bu}$ çalışma, tuz stresi koşulları altında 10 aspir çeşidinin çimlenme kapasitesini belirlemek için yapılmıștır. Beş farklı tuz $(\mathrm{NaCl})$ konsantrasyonu $(0$, $60,120,180,240 \mathrm{mM}$ ) aspir tohumlarının çimlenme kapasitesini test etmek için kullanılmıştır. Tohum çimlenmeleri 14 gün boyunca her gün sayılarak çimlenme yüzdesi, Timson's çimlenme indeksi, ortalama çimlenme zamanı, ortalama çimlenme oranı ve çimlenme stres endeksleri hesaplanmıştır. Çeşit ve tuz konsantrasyonlarının etkileri tüm çimlenme parametreleri için önemli olarak bulunurken, ortalama çimlenme zamanı ve ortalama çimlenme oranlarının interaksiyon etkileri önemsiz bulunmuştur. Çimlenme yüzdesi, Timson's çimlenme ve çimlenme stres indeksleri artan tuz konsantrasyonları ile önemli ölçüde azalmıştır. Ancak, yüksek tuz konsantrasyonu seviyelerinde ortalama çimlenme zamanı artmıştır. Çimlenme parametreleri arasında hesaplanan korelasyon katsayılarının tamamı önemli çıkmıştır. Çimlenme yüzdesindeki en önemli azalma 180 ve $240 \mathrm{mM}$ tuz konsantrasyonlarında gözlenmiştir. Çimlenme indeksi parametrelerine göre, Leed ve FO2 çeşitleri çimlenme aşamasında tuz stresine karşı daha hassas ve Royal çeşidi ise test edilen diğer çeşitlere göre daha dayanıklı olarak bulunmuştur. Çimlenme yüzdesi, Timson's çimlenme indeksi ve çimlenme stres endeksi tuz stresi altındaki aspir çeşitlerinde çimlenme kapasitesini değerlendirmek için uygun iken; ortalama çimlenme zamanı ve çimlenme oranı uygun bulunmamıştır. 


\section{Introduction}

Soil salinity is a major problem of agricultural lands, and it is caused by accumulation of salts in the soil through over watering and evaporation, irrigation with saline water and lack of adequate drainage systems. It is especially problematic in arid and semiarid parts of the world $[1,2]$. Soil salinization affects approximately 800 million hectares of agricultural land in the world. Over 1.5 million hectares of land is affected by salinity and alkalinity in Turkey [3].

Salinity affects many physiological processes in plants, such as sodium accumulation, reduction in water and nutrient absorption, production of reactive oxygen species leading to cellular damage, toxicity, water loss and decreased photosynthesis. As a result of these changes, plant growth is reduced and yields are decreased [4].

Safflower is an important oil seed crop and it has been grown for its olieferous seeds and flowers since ancient times. Safflower is cultivated more than 60 countries with production area of more than 650.000 hectares around the world. Turkey ranks as the 8th largest producer and the safflower is cultivated more than 15.000 hectares with over 21 million tons of seed production [5]. Oil demand and consumption exceeds the production in Turkey and oil deficit covered by import of oils and oliferous seeds. Therefore it is necessary to increase oil seed crop production in Turkey. In order to increase crop production, it is also necessary to alleviate the effects of environmental stress conditions on crops for increased yields [6].

Drought and salinity are the major causes of yield reduction in crop plants [2]. Therefore, it is necessary to investigate germination, growth and adaptation capabilities of crop plants required for food production under stress conditions. Safflower is known to be tolerant to drought and salinity stress and could be used as an alternative crop suitable to be grown under drought and saline conditions $[7,8]$. Application of stress conditions and their timing are important screening factors to determine tolerant and susceptible genotypes. Germination and seedling development phases of safflower are the most sensitive development periods to salinity stress [4]. Therefore, the aim of the present study was to evaluate diverse safflower cultivars under varying salt concentrations to determine their germination abilities and to calculate germination indices under salinity stress.

\section{Material and Method}

Seed of 10 safflower cultivars from 4 different countries were evaluated for their germination behaviors under different salt concentrations. Five safflower cultivars UC-1(PI 572434), Royal (PI 537694), US-10 (PI 572414), Leed (PI 572436) and Gila (PI 537692) were from United States, FO2 (PI 506426) was from China, Lesaf 414 (PI 603206) and AC Sunset (PI 592391) were from Canada, Quiriego 88 (PI 537110) and San Jose 89 (PI 561703) were from Mexico. Cultivars oil contents ranged from $25 \%$ to $31 \%$ in field trails and they were linoleic type with the exception of US-10, which was oleic type [9].

Seed surface sterilization was carried out by soaking seeds in 1\% sodium hypochlorite solution for $10 \mathrm{~min}$. After sterilization, all seeds were rinsed under running tap water for $5 \mathrm{~min}$ and dried at room temperature. Seeds were sown in $15 \mathrm{~cm}$ wide petri dishes and 5 different concentrations $(0,60,120,180$, $240 \mathrm{mM}$ ) of $\mathrm{NaCl}$ solution was added on filter papers. Control groups were wetted with distilled water. Germination tests were carried out in a germination cabinet under $25{ }^{\circ} \mathrm{C}$ for 14 days [10]. Each petri dish contained 40 seeds and germination tests were conducted with 3 replications. Number of germinated seeds was counted every day and germinated seeds were removed from petri dishes.

Germination data were logit transformed for normality of results. Germination percentage, mean germination time, Timson's germination index [11], mean germination rate [12] and germination stress index [13] were calculated according to formulas in Table 1. Results were subjected to analysis of variance (ANOVA) using IBM SPSS Statistics 22.0 software (SPSS Inc., Chicago, IL, USA). Duncan's multiple range test $(\mathrm{p} \leq 0.05)$ was used to discriminate the differences between the means. To show the relationship between measured parameters, Pearson linear correlation analysis (heatmap correlation) was calculated using OriginPro software (version 2021, OriginLab, Northampton, MA).

Table 1. Formulas used to calculate different germination parameters in the study

\begin{tabular}{lll}
\hline Parameter & Formula for calculation & Description \\
\hline Germination percentage & $\left(\mathrm{N}_{\mathrm{p}} / \mathrm{N}_{\mathrm{t}}\right) \times 100$ & $\begin{array}{l}\mathrm{N}_{\mathrm{p}}-\text { the number of germinated seeds, } \mathrm{N}_{\mathrm{t}}-\text { the total } \\
\text { number of seeds } \\
\mathrm{n}-\text { cumulative daily germination percentage for each day } \\
\text { of the test } \\
\text { di - day, ni - number of seeds germinated at day di, } \mathrm{N}- \\
\text { total number of seeds germinated in the test }\end{array}$ \\
Timson's germination index & $\Sigma \mathrm{n}$ & $\begin{array}{l}\mathrm{T}-\text { mean germination time } \\
\mathrm{S}_{\mathrm{i}}-\text { germination percentage under stress condition, } \mathrm{K}_{\mathrm{i}}-\end{array}$ \\
Mean germination rate & $\Sigma(\mathrm{ni} \times \mathrm{di}) / \mathrm{N}$ & germination percentage under non-stress condition \\
\hline
\end{tabular}




\section{Results}

Safflower cultivars were germinated under controlled conditions under 5 different salinity levels for 14 days and germination was recorded every day. Germination percentage and germination stress index were calculated at the end of the germination period. Daily counted germination values were used to calculate the other germination parameters. After germination tests, results were subjected to variance analysis and means were separated according to their importance levels. Germination results were used to calculate 5 germination parameters, and effects of cultivars, treatments and their interaction. Results of variance analysis were presented in Table 2. Cultivar and salt concentrations had significant effects on all germination indices $(\mathrm{P}<0.01)$. However interaction effects on mean germination time and mean germination rate was not important.

Germination percentage was higher for control groups for safflower cultivars. The lowest germination percentage was found in FO2 $(73.3 \%)$ and the highest germination percentage was observed in Leed (89.2\%) for the controls. Seeds subjected to germination test under different salt concentration exhibited reduced germination percentages than the control seeds. Increased salt concentrations from $60 \mathrm{mM}$ to $240 \mathrm{mM}$ caused reduced germination for all cultivars tested. Reduction in germination percentage was more severe for 180 and $240 \mathrm{mM}$ salt concentrations, hence these salt concentrations started to differ safflower cultivars for their germination ability under salt stress. Leed and F02 had the highest germination decline at 180 and $240 \mathrm{mM}$ salt concentrations (Table 3). Gila, UC-1 and Lesaf 414 showed significant decline for germination percentage for $240 \mathrm{mM}$ salt concentration as well.

Timson's germination index values based on germination data were similar to germination percentage. Higher germination index values were found for control seeds. As salt concentrations increased, germination index values started to decrease and the lowest germination index values were found in seeds germinated in $240 \mathrm{mM}$ salt concentrations (Table 3).

Mean germination times of cultivars belong to control group were generally lower than 2 days and only 4 cultivars had mean germination times higher than 2 days. Increased salt concentrations affected Leed, Royal, AC Sunset and FO2 mean germination times more those of other cultivars. Salt stress increased mean germination times of cultivars. Even though Royal and AC Sunset germination percentages were not greatly reduced by increased salt concentrations, their mean germination times increased as much as Leed and F02, and these cultivars had the highest increase in mean germination time in the study.

Mean germination rate ranged from 0.28 to 0.73 among the genotypes under different salt concentrations. Control groups had the highest mean germination rates in the study and the lowest mean germination rates were found in seeds germinated under high salt concentrations (Table 3).

Since control groups was not exposed salt stress conditions, it was not possible to calculate germination stress index for them. Seeds germinated under different salt concentrations showed different results for germination stress index depending on their germination capacity. Germination stress index was high at low salt concentrations; however, germination stress index values started to become lower as salt concentrations increased, showing germination of genotypes under salt stress was significantly affected by salt concentrations. The lowest germination stress index score was found in FO2 at $240 \mathrm{mM}$ salt concentration.

The result of the correlation analysis for germination parameters were given in Fig 1 . All the 10 coefficients are significant at the $p \leq 0.001$ levels. Among the 10 significant coefficients, 6 were positively and 4 were negatively correlated with each other. Germination percentage was positively correlated to Timson's germination index (0.98), mean germination rate (0.43) and germination stress index (0.93) and negatively correlated to Mean germination time (0.45).

\section{Discussion and Conclusion}

Salinization of agricultural soils is an important environmental stress factor that limit seed germination, plant growth and yield. In addition, salinization exacerbates soil conditions for plant growth by increasing soil sodium content and soil $\mathrm{pH}$, creating suboptimal conditions for plant growth and

Table 2. Variance analysis of cultivars, treatments and their interactions on germination parameters of safflower seeds

\begin{tabular}{ccccccc}
\hline & DF & \multicolumn{5}{c}{ Mean of squares } \\
\cline { 3 - 7 } & $\begin{array}{c}\text { Germination } \\
\text { percentage }\end{array}$ & $\begin{array}{c}\text { Timson's } \\
\text { germination index }\end{array}$ & $\begin{array}{c}\text { Mean germination } \\
\text { time }\end{array}$ & $\begin{array}{c}\text { Mean germination } \\
\text { rate }\end{array}$ & $\begin{array}{c}\text { Germination } \\
\text { stress index }\end{array}$ \\
\hline Cultivars & 9 & $427.77^{* *}$ & $128374.35^{* *}$ & $2.60^{* *}$ & $0.14^{* *}$ & $0.12^{* *}$ \\
Treatments & 4 & $2142.90^{* *}$ & $699040.73^{* *}$ & $4.20^{* *}$ & $0.21^{* *}$ & $4.09^{* *}$ \\
Interaction & 36 & $74.95^{* *}$ & $17853.73^{* *}$ & 0.09 ns & $0.005^{\text {ns }}$ & $0.031^{* *}$ \\
Eror & 100 & 11.46 & 2882.79 & 0.072 & 0.004 & 0.0001 \\
CV (\%) & & 5.97 & 7.87 & 12.34 & 13.32 & 1.98 \\
\hline
\end{tabular}


further reduces crop yields. Safflower and many other crop species are adversely affected by such conditions [14-17]. Different treatments were tested to alleviate these environmental conditions, such as chitosan application to seeds [18], seed priming [19] and foliar applications to plants [20]. Another way to increase crop productivity is to find suitable genotypes resistant to abiotic stress factors through screening [21, 22] and to incorporate necessary genes to elite lines through breeding [23].
In the present study, diverse safflower cultivars were screened for their germination capacities under 5 different salt concentrations. Different seed parameters were calculated using germination data. Seed germination parameters could be used to identify salinity tolerance of different genotypes at germination stage [24]. All tested cultivars had more than $50 \%$ germination up to $120 \mathrm{mM}$ salt concentration (Table 3). However, increasing salt concentration to 180 and $240 \mathrm{mM}$ differentiated

Table 3. The mean values of germination parameters in safflower cultivars under salt stress

\begin{tabular}{|c|c|c|c|c|c|c|}
\hline Genotype & $\begin{array}{c}\mathrm{NaCl} \\
\text { concentration } \\
(\mathrm{mM})\end{array}$ & $\begin{array}{l}\text { Germination } \\
\text { percentage }\end{array}$ & $\begin{array}{c}\text { Timson's } \\
\text { germination index }\end{array}$ & $\begin{array}{c}\text { Mean } \\
\text { Germination } \\
\text { Time }\end{array}$ & $\begin{array}{c}\text { Mean } \\
\text { germination } \\
\text { rate }\end{array}$ & $\begin{array}{l}\text { Germination } \\
\text { stress index }\end{array}$ \\
\hline \multirow{5}{*}{ Gila } & 0 (Control) & $85.8 \mathrm{a}-\mathrm{d}$ & 845.83 a-e & $2.15 \mathrm{j}-\mathrm{p}$ & $0.47 \mathrm{f}-\mathrm{m}$ & - \\
\hline & 60 & $77.5 \mathrm{~d}-\mathrm{i}$ & 737.50 e-n & $2.47 \mathrm{e}-\mathrm{l}$ & $0.41 \mathrm{i}-\mathrm{p}$ & $0.90 \mathrm{e}$ \\
\hline & 120 & $67.5 \mathrm{i}-\mathrm{n}$ & $639.17 \mathrm{k}-\mathrm{s}$ & $2.53 \mathrm{~d}-\mathrm{k}$ & $0.40 j-p$ & $0.79 \mathrm{k}-\mathrm{o}$ \\
\hline & 180 & 60.8 l-o & $574.17 \mathrm{o-u}$ & $2.58 \mathrm{c}-\mathrm{j}$ & 0.39 k-p & $0.71 \mathrm{~s}$ \\
\hline & 240 & $52.5 \mathrm{o}-\mathrm{s}$ & $473.33 \mathrm{uv}$ & $2.98 \mathrm{~b}-\mathrm{e}$ & $0.34 \mathrm{~m}-\mathrm{p}$ & $0.61 \mathrm{t}$ \\
\hline \multirow{5}{*}{ UC-1 } & 0 (Control) & $79.2 \mathrm{c}-\mathrm{h}$ & 831.67 a-f & $1.49 \mathrm{t}-\mathrm{y}$ & 0.67 a-c & - \\
\hline & 60 & $77.5 \mathrm{~d}-\mathrm{i}$ & 810.00 a-i & $1.55 \mathrm{~s}-\mathrm{y}$ & $0.66 \mathrm{a}-\mathrm{c}$ & $0.98 \mathrm{~b}$ \\
\hline & 120 & $66.7 \mathrm{j}-\mathrm{n}$ & 691.67 g-p & $1.62 \mathrm{p}-\mathrm{y}$ & 0.62 a-e & $0.84 \mathrm{~g}-\mathrm{i}$ \\
\hline & 180 & $60.0 \mathrm{~m}-\mathrm{p}$ & $601.67 n-u$ & $1.99 \mathrm{l}-\mathrm{u}$ & $0.52 \mathrm{e}-\mathrm{k}$ & $0.76 \mathrm{o}-\mathrm{r}$ \\
\hline & 240 & $49.2 \mathrm{r}-\mathrm{s}$ & $488.33 \mathrm{t}-\mathrm{v}$ & $2.06 \mathrm{j}-\mathrm{s}$ & $0.49 \mathrm{f}-\mathrm{l}$ & $0.62 \mathrm{t}$ \\
\hline \multirow{5}{*}{ Royal } & 0 (Control) & $87.5 \mathrm{ab}$ & $924.17 \mathrm{ab}$ & $1.43 \mathrm{vy}$ & $0.70 \mathrm{a}$ & - \\
\hline & 60 & $85.8 \mathrm{a}-\mathrm{d}$ & 895.83 a-c & $1.56 r-y$ & $0.65 \mathrm{a}-\mathrm{d}$ & $0.98 \mathrm{~b}$ \\
\hline & 120 & 84.2 a-e & 855.00 a-e & $1.84 \mathrm{n}-\mathrm{y}$ & $0.55 \mathrm{c}-\mathrm{h}$ & $0.96 \mathrm{~b}-\mathrm{d}$ \\
\hline & 180 & $75.8 \mathrm{e}-\mathrm{j}$ & $763.33 \mathrm{c}-\mathrm{l}$ & $1.94 \mathrm{~m}-\mathrm{v}$ & $0.52 \mathrm{e}-\mathrm{j}$ & $0.87 \mathrm{fg}$ \\
\hline & 240 & $71.7 \mathrm{~g}-\mathrm{l}$ & $660.00 \mathrm{j}-\mathrm{r}$ & $2.79 \mathrm{~b}-\mathrm{g}$ & $0.36 \mathrm{l}-\mathrm{p}$ & $0.82 \mathrm{~h}-\mathrm{k}$ \\
\hline \multirow{5}{*}{ US-10 } & 0 (Control) & 86.7 a-c & $920.00 \mathrm{ab}$ & $1.37 \mathrm{y}$ & $0.73 \mathrm{a}$ & - \\
\hline & 60 & $73.3 \mathrm{f}-\mathrm{k}$ & 773.33 c-k & $1.45 \mathrm{vy}$ & $0.69 \mathrm{ab}$ & $0.85 \mathrm{gh}$ \\
\hline & 120 & 70.8 h-m & 745.83 d-m & $1.47 \mathrm{u}-\mathrm{y}$ & $0.69 \mathrm{ab}$ & $0.82 \mathrm{~h}-\mathrm{l}$ \\
\hline & 180 & $67.5 \mathrm{i}-\mathrm{n}$ & 686.67 h-p & $1.83 \mathrm{n}-\mathrm{y}$ & $0.56 \mathrm{c}-\mathrm{g}$ & $0.78 \mathrm{~m}-\mathrm{p}$ \\
\hline & 240 & $66.7 \mathrm{j}-\mathrm{n}$ & $676.67 \mathrm{i}-\mathrm{r}$ & $1.85 \mathrm{n}-\mathrm{y}$ & $0.55 \mathrm{c}-\mathrm{h}$ & $0.77 n-p$ \\
\hline \multirow{5}{*}{ Leed } & 0 (Control) & $89.2 \mathrm{a}$ & $944.17 \mathrm{a}$ & $1.41 \mathrm{vy}$ & $0.71 \mathrm{a}$ & - \\
\hline & 60 & 70.0 h-n & $703.33 \mathrm{f}-\mathrm{o}$ & $2.02 \mathrm{k}-\mathrm{t}$ & $0.55 \mathrm{c}-\mathrm{h}$ & 0.79 l-o \\
\hline & 120 & 50.0 p-s & $486.67 \mathrm{uv}$ & $2.27 \mathrm{~g}-\mathrm{o}$ & $0.45 \mathrm{f}-\mathrm{n}$ & $0.56 \mathrm{u}$ \\
\hline & 180 & $31.7 \mathrm{t}$ & $306.67 \mathrm{y}$ & $2.32 \mathrm{f}-\mathrm{o}$ & 0.43 g-o & $0.36 \mathrm{v}$ \\
\hline & 240 & $28.3 \mathrm{t}$ & $262.50 \mathrm{y}$ & $2.77 \mathrm{~b}-\mathrm{h}$ & $0.36 \mathrm{l}-\mathrm{p}$ & $0.32 \mathrm{y}$ \\
\hline \multirow{5}{*}{ AC Sunset } & 0 (Control) & 84.2 a-e & 820.83 a-h & $2.24 \mathrm{~h}-\mathrm{o}$ & $0.45 \mathrm{f}-\mathrm{n}$ & - \\
\hline & 60 & $82.5 \mathrm{a}-\mathrm{f}$ & $790.83 \mathrm{~b}-\mathrm{j}$ & $2.39 \mathrm{f}-\mathrm{m}$ & 0.42 h-o & $0.98 \mathrm{~b}$ \\
\hline & 120 & 81.7 a-f & $746.67 \mathrm{~d}-\mathrm{m}$ & $2.79 \mathrm{~b}-\mathrm{g}$ & $0.36 \mathrm{l}-\mathrm{p}$ & $0.97 \mathrm{bc}$ \\
\hline & 180 & $68.3 \mathrm{i}-\mathrm{n}$ & $627.50 \mathrm{l}-\mathrm{s}$ & $2.84 b-f$ & $0.36 \mathrm{l}-\mathrm{p}$ & $0.81 \mathrm{i}-1$ \\
\hline & 240 & $61.7 \mathrm{l}$-o & $518.33 \mathrm{~s}-\mathrm{v}$ & $3.62 \mathrm{a}$ & $0.28 \mathrm{p}$ & $0.73 \mathrm{rs}$ \\
\hline \multirow{5}{*}{ Lesaf 414} & 0 (Control) & 85.0 a-d & 896.67 a-c & $1.46 \mathrm{u}-\mathrm{y}$ & $0.71 \mathrm{a}$ & - \\
\hline & 60 & 84.2 a-e & 884.17 a-c & $1.48 \mathrm{u}-\mathrm{y}$ & $0.70 \mathrm{a}$ & $0.99 \mathrm{a}$ \\
\hline & 120 & 80.8 b-g & $825.83 \mathrm{a}-\mathrm{g}$ & $1.780-y$ & $0.57 \mathrm{~b}-\mathrm{f}$ & $0.95 \mathrm{~b}-\mathrm{d}$ \\
\hline & 180 & 70.8 h-m & $698.33 \mathrm{f}-\mathrm{o}$ & $2.15 j-p$ & $0.47 \mathrm{f}-\mathrm{m}$ & $0.83 \mathrm{~h}-\mathrm{j}$ \\
\hline & 240 & $44.2 \mathrm{~s}$ & $431.67 \mathrm{v}$ & $2.22 \mathrm{i}-\mathrm{o}$ & $0.45 \mathrm{f}-\mathrm{n}$ & $0.52 \mathrm{u}$ \\
\hline \multirow{5}{*}{$\begin{array}{l}\text { Quiriego } \\
\quad 88\end{array}$} & 0 (Control) & 86.7 a-c & 880.83 a-d & $1.83 n-y$ & $0.55 \mathrm{c}-\mathrm{h}$ & - \\
\hline & 60 & $80.8 \mathrm{a}-\mathrm{g}$ & $816.67 \mathrm{a}-\mathrm{h}$ & $1.89 \mathrm{~m}-\mathrm{y}$ & $0.53 \mathrm{~d}-\mathrm{i}$ & $0.93 \mathrm{~d}$ \\
\hline & 120 & $70.0 \mathrm{~h}-\mathrm{n}$ & $700.83 \mathrm{f}-\mathrm{o}$ & $1.99 \mathrm{l}-\mathrm{u}$ & $0.50 \mathrm{e}-\mathrm{k}$ & $0.81 \mathrm{j}-\mathrm{m}$ \\
\hline & 180 & 65.0 k-n & $617.50 \mathrm{~m}-\mathrm{t}$ & $2.50 \mathrm{e}-\mathrm{l}$ & $0.40 \mathrm{i}-\mathrm{p}$ & $0.75 \mathrm{pr}$ \\
\hline & 240 & $60.8 \mathrm{l}-\mathrm{o}$ & $560.00 \mathrm{p}-\mathrm{v}$ & $2.80 \mathrm{~b}-\mathrm{g}$ & $0.36 \mathrm{l}-\mathrm{p}$ & $0.70 \mathrm{~s}$ \\
\hline \multirow{5}{*}{$\begin{array}{c}\text { San Jose } \\
89\end{array}$} & 0 (Control) & 82.5 a-f & 817.50 a-h & $2.09 \mathrm{j}-\mathrm{r}$ & $0.48 \mathrm{f}-\mathrm{l}$ & - \\
\hline & 60 & $73.3 \mathrm{f}-\mathrm{k}$ & 720.00 e-n & $2.18 \mathrm{i}-\mathrm{o}$ & $0.46 \mathrm{f}-\mathrm{n}$ & 0.89 ef \\
\hline & 120 & $67.5 \mathrm{i}-\mathrm{n}$ & 651.67 k-s & $2.36 \mathrm{f}-\mathrm{n}$ & 0.43 h-o & 0.82 h-l \\
\hline & 180 & $65.8 \mathrm{j}-\mathrm{n}$ & $624.17 \mathrm{~m}-\mathrm{s}$ & $2.52 \mathrm{~d}-\mathrm{l}$ & $0.40 \mathrm{i}-\mathrm{p}$ & 0.80 k-n \\
\hline & 240 & $59.2 \mathrm{n}-\mathrm{r}$ & $550.00 \mathrm{r}-\mathrm{v}$ & $2.71 \mathrm{c}-\mathrm{i}$ & $0.37 \mathrm{l}-\mathrm{p}$ & $0.72 \mathrm{~s}$ \\
\hline \multirow{5}{*}{ F02 } & 0 (Control) & $73.3 \mathrm{f}-\mathrm{k}$ & 728.33 e-n & $2.07 \mathrm{j}-\mathrm{s}$ & $0.49 \mathrm{f}-\mathrm{l}$ & - \\
\hline & 60 & 71.7 g-l & $702.50 \mathrm{f}-\mathrm{o}$ & $2.21 \mathrm{i}-\mathrm{o}$ & $0.46 \mathrm{f}-\mathrm{n}$ & $0.98 \mathrm{~b}$ \\
\hline & 120 & $69.2 \mathrm{~h}-\mathrm{n}$ & $620.83 \mathrm{~m}-\mathrm{s}$ & $3.03 \mathrm{~b}-\mathrm{d}$ & $0.33 n-p$ & $0.94 \mathrm{~cd}$ \\
\hline & 180 & $48.3 \mathrm{~s}$ & $431.67 \mathrm{v}$ & 3.08 bc & $0.33 \mathrm{n}-\mathrm{p}$ & $0.66 \mathrm{t}$ \\
\hline & 240 & $15.0 \mathrm{u}$ & $130.83 \mathrm{z}$ & $3.26 \mathrm{ab}$ & $0.31 \mathrm{op}$ & $0.20 \mathrm{z}$ \\
\hline
\end{tabular}




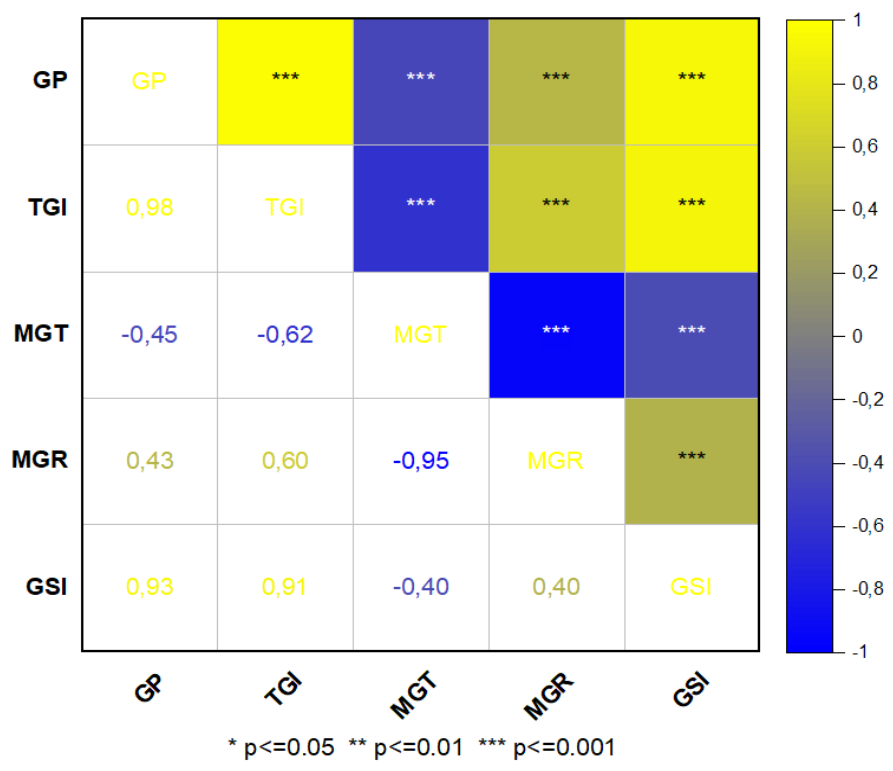

Figure 1. Relationships and correlation between germination parameters generated by Heat map using mean values of studied traits in safflower genotypes under salt. Color scale displays the intensity of normalized mean values of different parameters. (GP, Germination percentage; TGI, Timson's germination index; MGT, Mean germination time; MGR, Mean germination rate; GSI, Germination stress index)

cultivars based on their germination capacities. Leed (28.3\%), FO2 (15\%), Lesaf 414 (44.2\%) and UC-1 $(49.2 \%)$ had lower than $50 \%$ germination at $240 \mathrm{mM}$ salt concentration. The other safflower cultivars all had higher than $50 \%$ germination percentages.

Timson's germination index uses daily cumulative germination percentage and the higher value of Timson's index correlates to speedy germination. Consequently, control seeds, which have not been exposed to salt stress, had higher values for Timson's index, hence speedier germination as observed by mean germination times as well. As germination percentage decreased and mean germination time increased with higher salt concentrations, Timson's germination index, speed of germination, decreased. The lowest values for Timson's germination index was found for F02 and Leed, which had the lowest germination percentages and high mean germination times at $240 \mathrm{mM}$ salt concentrations.

Mean germination time is another germination parameter used to calculate relative germination speed. It is used to compare effects of different treatments on speed of germination but it does not always correlate with time to germinate to reach a specific germination percentage [25]. Increased salt concentrations caused an increase in mean germination times of cultivars. However, mean germination times of FO2 (3.26) and Leed (2.77) was lower than AC Sunset (3.62) at $240 \mathrm{mM}$ salt concentration, whose germination percentage was higher than FO2 and Leed. Mean germination time did not correspond to germination percentages at $240 \mathrm{mM}$ salt concentration may have overestimated speed of germination in FO2 and Leed. Similarly, San Jose 89 had high mean germination time at $0 \mathrm{mM}$ but mean germination time increase for $240 \mathrm{mM}$ was 0.62 , lower than Royal's mean germination time.

Mean germination rate is based on mean germination time and they have inverse relationship to each other. As mean germination time is higher, mean germination rate gets lower. Consequently, higher mean germination rates were observed in control seeds, but mean germination rate values did not correspond to germination percentages. Both San Jose 89 and Lesaf 414 had similar germination percentages for their controls, their mean germination rates were very different (Table 3 ) due to differences in mean germination times, and negative correlation $(-0.95)$ observed between the two parameters (Table 3 ).

Germination stress index was also calculated to determine effects of salt stress on germination capacity of seeds. Leed showed 29\% decrease in germination stress index from $60 \mathrm{mM}$ to $120 \mathrm{mM}$ salt concentration. The highest decreases after Leed at the same salt concentrations was observed in Quiriego 88 and Gila by $13 \%$ and $12 \%$; respectively. At 180 and $240 \mathrm{mM}$ salt concentrations, both Leed and FO2 had the lowest germination stress index values compared to other genotypes. Based on germination stress index, Leed was more susceptible than FO2 and Royal was the most tolerant cultivar to salt stress at germination stage.

Based on germination indices, it could be concluded that the germination percentage, Timson's germination index and germination stress index were the best indices for the assessment of the germination capacity compared to mean germination time and mean germination rate. These two indices 
might be affected by the seed quality and seed features, such as imbibition time. These indices might reflect differences related to those characters rather than the differences in germination capacity under stress conditions. Based on these indices, Leed and F02 were sensitive and Royal and US-10 were resistant to salt stress at the germination stage.

\section{Acknowledgment}

Sercan Önder and Murat Mutlucan were financially supported by Council of Higher Education under YÖK $100 / 2000$ fellowship program for graduate students.

\section{Declaration of Ethical Code}

In this study, we undertake that all the rules required to be followed within the scope of the "Higher Education Institutions Scientific Research and Publication Ethics Directive" are complied with, and that none of the actions stated under the heading "Actions Against Scientific Research and Publication Ethics" are not carried out.

\section{References}

[1] Ekmekçi, E., Apan, M., Kara, T. 2005. Tuzluluğun Bitki Gelişimine Etkisi. Ondokuz Mayıs Üniversitesi Ziraat Fakültesi Dergisi, 20(3), 118125.

[2] Çulha, Ş., Çakırlar, H. 2011. Tuzluluğun Bitkiler Üzerine Etkileri ve Tuz Tolerans Mekanizmaları. Afyon Kocatepe Üniversitesi Fen Bilimleri Dergisi, 11 (2), 11-34.

[3] Türkan, I., Demiral, T. 2009. Recent Developments in Understanding Salinity Tolerance. Environmental and Experimental Botany, 67(1), 2-9.

[4] Hussain, M. I., Lyra, D. A., Farooq, M., Nikoloudakis, N., Khalid, N. 2016. Salt and Drought Stress in Safflower: A Review. Agronomy and Sustainable Development, 36(1), 1-31.

[5] Food and Agriculture Organization (FAO), 2021. Statistical Data for Safflower Agriculture. http://www.fao.org/faostat/en/\#data/QC (Erişim Tarihi: 20.05.2021).

[6] Frona, D., Szenderak, J., Harangi-Rakos, M. 2019. The Challenge of Feeding the World. Sustainability, 11(20), 5816.

[7] Baydar, H., Turgut İ. 1992. Aspir (Carthamus tinctorius L.)'in Antalya Koşullarında Kışlık Olarak Yetiştirme Olanakları. Akdeniz Üniversitesi Ziraat Fakültesi Dergisi, 5(1-2):7592.

[8] Beg, A. 1994. Status and Potential of Some Oilseed Crops in the WANA Region.
International Center for Agricultural Research in the Dry Areas (ICARDA), 6-8 July, Aleppo, 38.

[9] Erbaş, S., Tonguç, M., Şanlı, A. 2016. Variations in the Agronomic and Quality Characteristics of Domestic and Foreign Safflower (Carthamus tinctorius L.) Genotypes. Turkish Journal of Field Crops, 21(1), 110-119.

[10] ISTA, (2009). International Rules for Seed Testing. International Seed Testing Association, Bassersdorf.

[11] Al-Ansari, F., Ksiksi, T. 2016. A Quantative Assessment of Germination Parameters: The Case of Crotalaria persica and Tephrosia apollinea. The Open Ecology Journal, 9(1), 13-21.

[12] Ranal, M. A., Santana, D. G. D., Ferreira, W. R., Mendes-Rodrigues, C. 2009. Calculating Germination Measurements and Organizing Spreadsheets. Brazilian Journal of Botany, 32(4), 849-855.

[13] Bouslama, M., Schapaugh, W. T. (1984). Stress Tolerance in Soybeans. I. Evaluation of Three Screening Techniques for Heat and Drought Tolerance. Crop Science, 24(5), 933-937.

[14] Irwing, D. W., Shannon, M. C., Breda, V. A., Mackey, B. E. 1988. Salinity Effects on Yield and Oil Quality of High-Linoleate and High-Oleate Cultivars of Safflower (Carthamus tinctorius L.). Journal of Agricultural and Food Chemistry, 36(1), 37-42.

[15] Gadallah, M. A. A., Ramadan, T. 1997. Effects of Zinc and Salinity on Growth and Anatomical Structure of Carthamus tinctorius L. Biologia Plantarum, 39(3), 411-418.

[16] Kaya, M. D, İpek, A., Öztürk, A. 2003. Different Soil Salinity Levels on Germination and Seedling Growth of Safflower (Carthamus tinctorius L.). Turkish Journal of Agriculture and Forestry, 27(4), 221-227.

[17] Çulha, Ş., Çakırlar, H. 2011. Effects of Salt Stress Induced by $\mathrm{NaCl}$ on Safflower (Carthamus tinctorius L.) Cultivars at Early Seedling Stages. Hacettepe Journal of Biology and Chemistry, 39(1), 61-64.

[18] Gürsoy, M. 2020. Effect of Chitosan Pretreatment on Seedling Growth and Antioxidant Enzyme Activity of Safflower (Carthamus tinctorius L.) Cultivars Under Saline Conditions. Applied Ecology and Environmental Research, 18(5), 6589-6603.

[19] Ashrafi, E., Razmjoo, J. 2015. Seed Treatment to Overcome Salt and Drought Stresses During Germination in Safflower (Carthamus tinctorius L.). Journal of Plant Nutrition, 38(14), 21512158.

[20] Jabeen, N., Ahmad, R. 2012. Improving Tolerance of Sunflower and Safflower During Growth 
Stages to Salinity Through Foliar Spray of Nutrient Solutions. Pakistan Journal of Botany, 44(2), 563-572.

[21] Jabeen N, Ahmad R. 2013. Variations in accessions of sunflower and safflower under stress conditions. Pakistan Journal of Botany, 45(2), 383-390.

[22] Kaya, M. D., Akdoğan, G., Kulan, E. G., Dağhan, H., Sarı, A. 2019. Salinity Tolerance Classification of Sunflower (Helianthus annuus L.) and Safflower (Carthamus tinctorius L.) by Cluster and Principal Component Analysis. Applied Ecology and Environmental Research, 17(2), 3849-3857.

[23] Pahlavani, M. H., Saeidi, G., Mirlohi, A. F. 2006.
Estimates of Genetic Parameters for Seed Germination of Safflower in Different Salinity Levels. Asian Journal of Plant Sciences, 5(1), 133-138.

[24] Aflaki, F., Sedghi, M., Pazuki, A., Pessarakli, M. 2017. Investigation of Seed Germination Indices for Early Selection of Salinity Tolerant Genotypes: A Case Study in Wheat. Emirates Journal of Food and Agriculture, 29(3), 222-226.

[25] Soltani, E., Ghaderi-Far, F., Baskin, C. C., Baskin, J. M. 2015. Problems with Using Mean Germination Time to Calculate Rate of Seed Germination. Australian Journal of Botany, 63(8), 631-635. 